\title{
On the Hausdorff dimension of piecewise hyperbolic attractors
}

\author{
by
}

\author{
Tomas Persson (Warszawa)
}

\begin{abstract}
We study non-invertible piecewise hyperbolic maps in the plane. The Hausdorff dimension of the attractor is calculated in terms of the Lyapunov exponents, provided that the map satisfies a transversality condition. Explicit examples of maps for which this condition holds are given.
\end{abstract}

1. Introduction. A general class of piecewise hyperbolic maps was studied by Pesin in [10]. Pesin proved the existence of SRB-measures and investigated their ergodic properties. Results from Pesin's article and Sataev's article [11] are described in Section 3. The assumptions in [10] and [11] did not allow overlaps of the images. Schmeling and Troubetzkoy extended in [12] the theory in [10] to allow maps with overlapping images.

Using the results of Pesin and techniques from Solomyak's paper [14, the author of this paper proved in [8] and [9] that for two classes of piecewise affine hyperbolic maps, there exists, for almost all parameters, an invariant measure that is absolutely continuous with respect to Lebesgue measure, provided that the map expands area. This result had previously been obtained for fat baker's transformations by Alexander and Yorke in [1]. The main difficulty that arises for the class of maps in [9] is that the symbolic space associated to the systems changes with the parameters, and also the SRB-measure changes in a way that is hard to control. By embedding all symbolic spaces into a larger space it was possible get sufficient control to prove the result.

Solomyak's proof in [14 uses a transversality property of power series. The proofs in [8] and [9] use the fact that iterates of points can be written as power series with such a transversality property. For the iterates to be expressible as power series, it is important that the directions of contraction are mapped onto each other throughout the manifold. The method in [8] and 
[9] is therefore not good for proving similar results for more general maps. It should also be noted that this method only gives results that hold for almost every map, with respect to some parameter.

Tsujii studied in [15] a class of area-expanding solenoidal attractors and proved that generically these systems have an invariant measure that is absolutely continuous with respect to Lebesgue measure. Tsujii also used a transversality condition, but in a different way. Instead of transversality of power series, Tsujii used transversality of intersections of iterates of curves. This technique makes it possible to show the existence of an absolutely continuous invariant measure for a fixed system, provided that an appropriate transversality condition is satisfied. Tsujii proved that this condition is generically satisfied.

In this paper we will use the idea from Tsujii's article 15] to prove a formula for the dimension of the attractor for some piecewise hyperbolic maps in the plane, provided that a transversality condition is satisfied. This is done by estimating the dimension from below. The estimate coincides with the previously known estimate from above (see [5] and [12]) and thereby provides the following formula for the dimension:

$$
\operatorname{dim}_{\mathrm{H}} \Lambda=1-\frac{\chi_{\mathrm{u}}}{\chi_{\mathrm{s}}},
$$

where $\Lambda$ denotes the attractor and $\chi_{\mathrm{u}}$ and $\chi_{\mathrm{s}}$ denote the positive and the negative Lyapunov exponents. This formula has previously been proved by Falconer in [5] and by Simon in [13], but for a much smaller class of systems. Both Falconer and Simon considered maps that are skew-products with the underlying shift being a full shift on $n$ symbols. These restrictions are not assumed in this paper. Hence, this paper generalises the results of Falconer and Simon.

For technical reasons in the proof, we will also need the assumption that the multiplicity entropy is zero, which is also assumed in Falconer's and Simon's results. This seems often to be the case, and we provide a condition which guarantees that the multiplicity entropy is zero. The author of this paper suspects that the condition of zero multiplicity entropy is not needed, but have not been able to prove the result without this condition. Instead we give conditions (see Lemma 2) that imply zero multiplicity entropy.

2. Outline of the paper. In Section 3 we describe the class of piecewise hyperbolic maps that is studied in this paper. In Section 4 we introduce a transversality condition. Under the assumption that this transversality condition holds and that the multiplicity entropy is zero, a theorem that estimates the dimension from below is stated in Section 5. This estimate gives the dimension formula. The theorem is proved in Section 8 . 
Section 6 contains a condition that guarantees zero multiplicity entropy, and Section 7 contains explicit examples of maps that satisfy the assumptions of this paper. There are also examples that the dimension formula may fail if the transversality condition does not hold.

3. Piecewise hyperbolic maps. In this paper we study maps on open and bounded subsets of the plane that are piecewise continuous and hyperbolic. Under certain conditions, stated later in this section, we will provide a dimension formula for the attractor of the map. This dimension formula is already known in the case where the map is injective, so in this paper the case of non-injective maps is considered.

The underlying theory of this paper is based on [10] and [12. In the first of these papers, Pesin studied a class of piecewise hyperbolic and injective maps on some manifold. This theory was extended to non-injective maps by Schmeling and Troubetzkoy in [12].

The conclusions of the papers [10] and [12] are that there is an attractor that can be decomposed into ergodic components such that on each component there is an ergodic measure, called an SRB-measure, with the property that the set of typical points has positive Lebesgue measure and the conditional measures on unstable manifolds are absolutely continuous with respect to the one-dimensional Lebesgue measure. Sataev showed in [11] that there are only finitely many ergodic components.

Let us now state the assumptions of this paper. The following assumptions (A1)- A4 are similar to the assumptions in [12, but in some cases more restrictive. In particular we only consider two-dimensional maps. For the more general setting we refer to [12] and [10].

Consider the plane $\mathbb{R}^{2}$ with the usual metric $d$. Let $K \subset \mathbb{R}^{2}$ be an open, bounded and connected set and let $N \subset K$ be a closed set in $K$. The set $N$ is called the discontinuity set. Let $f: K \backslash N \rightarrow K$.

Put

$$
\begin{aligned}
K^{+} & =\left\{x \in K: f^{n}(x) \notin N \cup \partial K, n=0,1,2, \ldots\right\}, \\
D & =\bigcap_{n \in \mathbb{N}} f^{n}\left(K^{+}\right) .
\end{aligned}
$$

The attractor of $f$ is the set $\Lambda=\bar{D}$.

The first condition is

(A1) the set $K \backslash N$ can be decomposed into finitely many open sets $K_{i}$ such that $f: K_{i} \rightarrow f\left(K_{i}\right)$ can be extended to a $C^{2}$-diffeomorphism from $\bar{K}_{i}$ to $\overline{f\left(K_{i}\right)}$.

Note that we only assume that $f$ restricted to $K_{i}$ is a diffeomorphism, so that the images $f\left(K_{i}\right)$ may intersect. 
For the next assumption we need some more notations. Let

$$
\begin{aligned}
& N^{+}=N \cup \partial K, \\
& N^{-}=\left\{y \in K: \exists z_{n} \in K \backslash N^{+}, z \in N^{+} \text {such that } z_{n} \rightarrow z, f\left(z_{n}\right) \rightarrow y\right\} .
\end{aligned}
$$

One might want to think of $N^{-}$as the image of $N^{+}$although $f$ is not defined on $N^{+}$. For $\varepsilon>0$ and $l=1,2, \ldots$, let

$$
\begin{aligned}
D_{\varepsilon, l}^{+} & =\left\{x \in K^{+}: d\left(f^{n}(x), N^{+}\right) \geq l^{-1} e^{-\varepsilon n}, n \in \mathbb{N} \cup\{0\}\right\} \\
D_{\varepsilon, l}^{-} & =\left\{x \in \Lambda: \exists\left(x_{n}\right)_{n=-\infty}^{0}, f\left(x_{n}\right)=x_{n+1}, x_{0}=x,\right. \\
& \left.d\left(x_{-n}, N^{-}\right) \geq l^{-1} e^{-\varepsilon n}, n \in \mathbb{N} \cup\{0\}\right\}, \\
D_{\varepsilon, l}^{0} & =D_{\varepsilon, l}^{+} \cap D_{\varepsilon, l}^{-}, \\
D_{\varepsilon}^{0} & =\bigcup_{l \geq 1}\left(D_{\varepsilon, l}^{+} \cap D_{\varepsilon, l}^{-}\right) .
\end{aligned}
$$

The hyperbolicity of the map $f$ is described in the next assumption:

(A2) There exists $C>0$ and $0<\lambda<1$ such that for every $x \in K \backslash N^{+}$ there exist cones $C^{\mathrm{s}}(x), C^{\mathrm{u}}(x) \subset T_{x} M$ such that the angle between $C^{\mathrm{s}}(x)$ and $C^{\mathrm{u}}(x)$ is uniformly bounded away from zero. For every $i$,

$$
\begin{array}{ll}
d_{x} f\left(C^{\mathrm{u}}(x)\right) \subset C^{\mathrm{u}}(f(x)), & \forall x \in K \backslash N, \\
d_{x}\left(f_{i}^{-1}\right)\left(C^{\mathrm{s}}(x)\right) \subset C^{\mathrm{s}}\left(f_{i}^{-1}(x)\right), & \forall x \in f\left(K_{i}\right),
\end{array}
$$

where $f_{i}$ denotes the restriction of $f$ to $K_{i}$, and for any $n>0$,

$$
\begin{array}{ll}
\left\|d_{x} f^{n}(v)\right\| \geq C \lambda^{-n}\|v\|, & \forall x \in K^{+}, \forall v \in C^{\mathrm{u}}(x), \\
\left\|\left(d_{x} f^{n}\right)^{-1}(v)\right\| \geq C \lambda^{-n}\|v\|, & \forall x \in K^{+}, \forall v \in C^{\mathrm{s}}\left(f^{n}(x)\right) .
\end{array}
$$

The cone families $C^{\mathrm{u}}(x)$ and $C^{\mathrm{s}}(x)$ depend continuously on $x \in K_{i}$ and they can be extended continuously to the boundary.

This assumption makes it possible to define stable and unstable manifolds, $W^{\mathrm{s}}(x)$ and $W^{\mathrm{u}}(x)$, as well as local ones for any $x \in D_{\varepsilon}^{0}$. It is then of course natural to ask whether $D_{\varepsilon}^{0}$ is empty or not. Under the assumptions of this paper it is shown in [10] that the set $D_{\varepsilon}^{0}$ is not empty for sufficiently small $\varepsilon>0$. (Here sufficiently small means so small that there are local unstable manifolds.)

We will also need the following assumption on the regularity of the boundary of $K_{i}$.

(A3) The sets $\partial K$ and $N$ are unions of finitely many $C^{2}$ curves such that the angle between these curves and the unstable cones are bounded away from zero, at any point of the closure of $f(K \backslash N)$.

Note that we do not assume anything about the union of the curves in (A3); the curves are allowed to intersect. 
3.1. Multiplicity entropy. Let $\mathcal{K}=\left\{K_{1}, \ldots, K_{p}\right\}$ be the partition of $K$ into sets on which $f$ is continuous, and let $\mathcal{K}_{n}$ be the corresponding partition for the map $f^{n}$. Let $k_{n}$ be the maximal numbers of elements of $\mathcal{K}_{n}$ that meet in one point. The multiplicity entropy $h_{\text {mult }}(f)$ is defined as

$$
h_{\text {mult }}(f)=\limsup _{n \rightarrow \infty} \frac{1}{n} \log k_{n} .
$$

Note that if $f$ satisfies assumptions A1 - A3 , then $f^{n}$ need not satisfy (A3). For $k_{n}$ to be finite, it is however only necessary that $f$ satisfies (A3). See [12] for details.

We can now give our next assumption:

$$
h_{\text {mult }}(f)=0 .
$$

This condition is stronger than the corresponding conditions in [10] and [12]. The assumptions used in these papers are essentially that the mulitiplicity entropy is smaller than the positive Lyapunov exponent. We will need this stronger version due to technical reasons in the proof.

One might wonder how general the condition $(\mathrm{A} 4 \mathrm{l})$ is. The author of this paper believes that this condition is, in some sense, easily satisfied. However there are examples of maps satisfying (A1)-(A3) such that the multiplicity entropy is larger than the positive Lyapunov exponent. In Section 6 we give sufficient conditions for a map to satisfy (A4). These conditions are for instance satisfied by Belykh maps (see [12]). Hence Belykh maps have zero multiplicity entropy.

We note that, in case $f$ is piecewise affine, condition (A4) implies that the topological entropy is equal to the entropy of the SRB-measure. This follows from the fact that the entropy of the SRB-measure is equal to the positive Lyapunov exponent (see [10]), and the result of Kruglikov and Rypdal in [7] that $h_{\mathrm{top}} \leq \chi_{\mathrm{u}}+h_{\mathrm{mult}}$ for piecewise affine maps (in the case of a map on the plane with one positive and one negative Lyapunov exponent; the statement in [7] is for any dimension).

4. A transversality condition. Let $\varepsilon>0$ and $0<\delta<1$. We will say that an intersection of two smooth curves $\gamma_{1}$ and $\gamma_{2}$ is $(\varepsilon, \delta)$-transversal if for any ball $B_{\varepsilon}$ of radius $\varepsilon$ intersecting both $\gamma_{1}$ and $\gamma_{2}$, there exist $x_{1} \in B_{\varepsilon} \cap \gamma_{1}$ and $x_{2} \in B_{\varepsilon} \cap \gamma_{2}$ such that the following holds true. If $d_{1}$ and $d_{2}$ are the induced metrics on $\gamma_{1}$ and $\gamma_{2}$ respectively, then the intersection of the open sets

$$
\bigcup_{y \in \gamma_{i} \cap B_{\varepsilon}} B\left(y, \delta d_{i}\left(x_{i}, y\right)\right), \quad i=1,2,
$$

is empty. The symbol $B(x, r)$ denotes the open ball of radius $r$ around $x$. Note that if $\gamma_{1}$ and $\gamma_{2}$ intersect $(\varepsilon, \delta)$-transversally then $\gamma_{1} \cap \gamma_{2}$ can be empty. 
Definition 1. We will say that a piecewise hyperbolic system $f: K \backslash N$ $\rightarrow K$ satisfies condition (T) if

(T) there exist $\varepsilon, \delta>0$ such that if $\gamma_{1}$ and $\gamma_{2}$ are two smooth curves such that every tangent lies in the unstable cone, and $\gamma_{1}$ and $\gamma_{2}$ are in different $K_{i}$, then the curves $f\left(\gamma_{1}\right)$ and $f\left(\gamma_{2}\right)$ intersect $(\varepsilon, \delta)$ transversally.

5. Dimension of the attractor. Consider a map $f: K \backslash N \rightarrow K \subset \mathbb{R}^{2}$ that satisfies conditions A1 -A4). We denote by $\chi_{\mathrm{s}}(x)<0<\chi_{\mathrm{u}}(x)$ the two Lyapunov exponents at the point $x$ if they exist. If $\Lambda_{1}$ is an ergodic component of the attractor, then the Lyapunov exponents are constant almost everywhere with respect to the SRB-measure on $\Lambda_{1}$, and we write $\chi_{\mathrm{s}}(x)=\chi_{\mathrm{s}}$ and $\chi_{\mathrm{u}}(x)=\chi_{\mathrm{u}}$ for almost every $x$. With these notations we have the following theorem.

Theorem 1. Suppose that $f: K \backslash N \rightarrow K \subset \mathbb{R}^{2}$ is a piecewise hyperbolic map that satisfies conditions (T) and (A1)- A4). Let $\Lambda_{1}$ be an ergodic component of the attractor, with one positive and one negative Lyapunov exponent, $\chi_{\mathrm{u}}$ and $\chi_{\mathrm{s}}$. Then the Hausdorff dimension of $\Lambda_{1}$ satisfies

$$
\operatorname{dim}_{\mathrm{H}} \Lambda_{1} \geq \min \left\{2,1-\chi_{\mathrm{u}} / \chi_{\mathrm{s}}\right\} .
$$

Theorem 1 is proved in Section 8 .

Note that in [12] it is proved that $\operatorname{dim}_{\mathrm{H}} \Lambda_{1} \leq 1-\chi_{\mathrm{u}} / \chi_{\mathrm{s}}$ with equality if and only if $f$ restricted to $\Lambda_{1}$ is almost everywhere invertible, meaning that $f$ is invertible on a set of full SRB-measure. Hence we get the following corollary.

COROLlary 1. If the assumptions of Theorem 1 are satisfied then

$$
\operatorname{dim}_{\mathrm{H}} \Lambda_{1}=\min \left\{2,1-\chi_{\mathrm{u}} / \chi_{\mathrm{s}}\right\},
$$

and $f$ is invertible almost everywhere on $\Lambda_{1}$ if and only if $\chi_{\mathrm{u}}+\chi_{\mathrm{s}} \leq 0$.

REMARK 1. In case the transversality condition $(T)$ is not satisfied we can only give the trivial estimate $\operatorname{dim}_{\mathrm{H}} \Lambda_{1} \geq 1$. Indeed, the map $f:[0,1]^{2} \rightarrow$ $[0,1]^{2}$ defined by $f:\left(x_{1}, x_{2}\right) \mapsto\left(x_{1} / 2,2 x_{2} \bmod 1\right)$ has the attractor $\Lambda_{1}=$ $\left\{\left(x_{1}, x_{2}\right): x_{1}=0,0 \leq x_{1} \leq 1\right\}$, and so $\operatorname{dim}_{\mathrm{H}} \Lambda_{1}=1$. Moreover, any map satisfying (A1) A4 has an attractor $\Lambda_{1}$ that contains curves of unstable manifolds. This implies that $\operatorname{dim}_{\mathrm{H}} \Lambda_{1} \geq 1$. So, unless one imposes an additional condition, such as $(\mathrm{T})$, one cannot get a better estimate than $\operatorname{dim}_{H} \Lambda_{1} \geq 1$. It should be noted that there are no obvious reasons why (A4) should be needed. It is here because of technical reasons in the proof.

6. Vanishing multiplicity entropy. In this section we give a condition which guarantees that the multiplicity entropy is zero. 
Theorem 2. Let $K, N \subset \mathbb{R}^{2}$ where $N$ is a union of smooth curves, and let $f: K \backslash N \rightarrow K$ satisfy conditions A1 Assume that there is a family of cones $C^{\mathrm{d}}(p, \gamma) \subset T_{p} \mathbb{R}^{2}$, where $p$ is a point on a smooth curve $\gamma \subset N$, such that $C^{\mathrm{d}}(p, \gamma)$ contains the tangents of $\gamma$ at $p$,

$$
C^{\mathrm{d}}(p, \gamma) \cap C^{\mathrm{u}}(p)=\{0\} \quad \text { and } \quad d f\left(C^{\mathrm{d}}\right) \subset C^{\mathrm{u}} .
$$

Then the multiplicity entropy of $f$ is zero.

REMARK 2. The condition $C^{\mathrm{d}}(p, \gamma) \cap C^{\mathrm{u}}(p)=\{0\}$ in Theorem 2 is formally nonsense since $C^{\mathrm{u}}(p)$ is not defined for $p \in N$. But $C^{\mathrm{u}}(p)$ depends continuously on $p \in K_{i}$ so the condition should be be understood as concerning an appropriate limit for each $K_{i}$ that meets $p$. This can be done by $\mathrm{A} 2$.

REMARK 3. It should be noted that if $f$ satisfies the conditions in Theorem 2, then so does any sufficiently small smooth perturbation of $f$.

Proof. For simplicity, let us start with the case that the curves of $N$ do not intersect. Let $p \in N$. We will iterate $p$ and see into how many pieces a small neighbourhood $U$ of $p$ is cut by a curve in $N$ that goes through $f^{n}(p)$. Of course, $f^{n}(p)$ is not defined but we will use this notation, for simplicity, for the collection of accumulation points of $f^{n}(q)$ as $q \rightarrow p$.

In the first iterate $U$ is cut through $p$ into at most two pieces, which we denote by $U_{1}$ and $U_{2}$ (or just $U_{1}$ if $U$ is not cut). In the next iterate, each

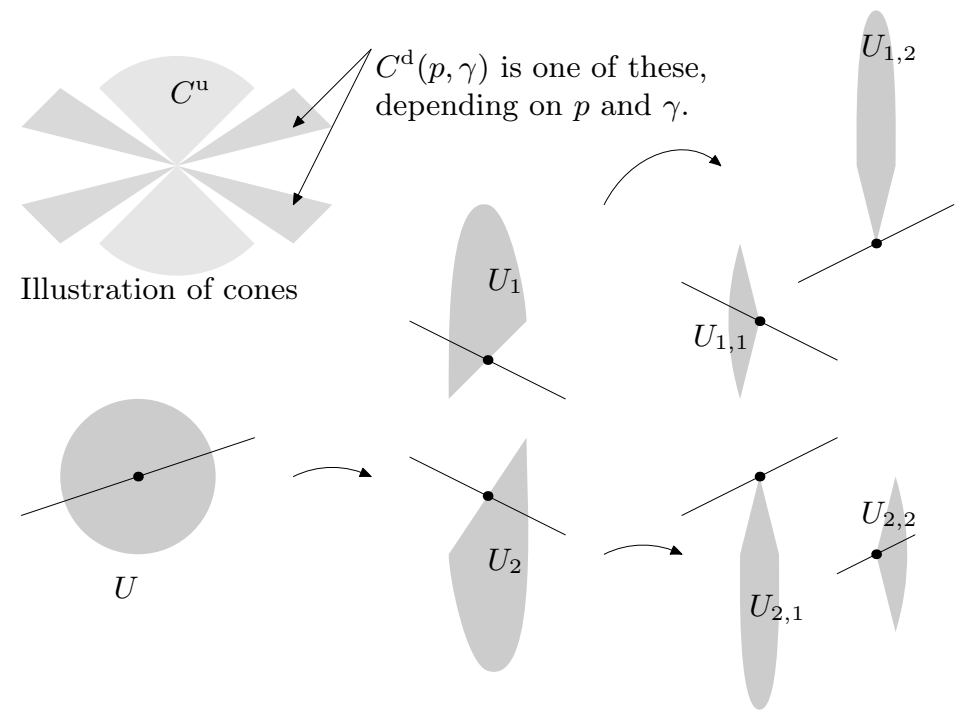

Fig. 1. Illustration to the proof of Theorem 2 Note that $U_{1,2}$ and $U_{2,1}$ cannot be cut through $f^{2}(p)$ since the slopes of the discontinuities are too small. In this example $C^{\mathrm{d}}(p, \gamma)$ takes two different forms, depending on $p$ and $\gamma$, as is shown in the figure. 
of the pieces $U_{1}$ and $U_{2}$ is cut through $f(p)$ into at most two pieces. Denote by $U_{1,1}$ and $U_{1,2}$ the pieces of $U_{1}$ and similarly for $U_{2}$.

By the property (2), one of $U_{1,1}$ and $U_{1,2}$ lies in the cone $C^{\mathrm{u}}\left(f^{2}(p)\right)$ and no iterate of this piece will be cut through $f^{n}(p)$ for any $n$. The same argument holds for the pieces $U_{2,1}$ and $U_{2,2}$. So we now have at most four pieces of which at most two can be cut in future iterations. There is a picture of this in Figure 1 .

By induction we find that after $n$ iterates $f^{n}(U)$ consists of at most $2 n$ pieces. This shows that the multiplicity entropy is zero.

The case of $N$ containing curves that cut each other is similar. If at most $L$ curves meet in one point, we deduce that after $n$ iterates, $U$ consists of at most $2(L+1) n$ pieces of which at most two can be cut through $f^{n}(p)$.

7. An example. In this section we give an example of maps satisfying the assumptions of Theorem 1 .

Let $K=(-1,1) \times(-1,1)$ be a square. Take $-1<k<1$ and let $N=$ $\left\{\left(x_{1}, x_{2}\right) \in K: x_{2}=k x_{1}\right\}$ be the singularity set. Take $\rho \neq 0$ and let $\psi_{1}$ and $\psi_{2}$ be two $C^{2}$ functions such that $\left|\psi_{1}^{\prime}\right|,\left|\psi_{2}^{\prime}\right|<\rho_{\psi}<|\rho| / 2$. We take parameters $1 / 2<\lambda<1,1<\gamma<2, a_{1}, a_{2}, b_{1}$ and $b_{2}$ such that the map $f$ defined by

$$
f\left(x_{1}, x_{2}\right)= \begin{cases}\left(\lambda x_{1}+a_{1}+\rho x_{2}+\psi_{1}\left(x_{2}\right), \gamma x_{2}+b_{1}\right) & \text { if } x_{2}>k x_{1}, \\ \left(\lambda x_{1}+a_{2}+\psi_{2}\left(x_{2}\right), \gamma x_{2}+b_{2}\right) & \text { if } x_{2}>k x_{1},\end{cases}
$$

maps $K \backslash N$ into $K$. There is a picture of $f$ in Figure 2 .

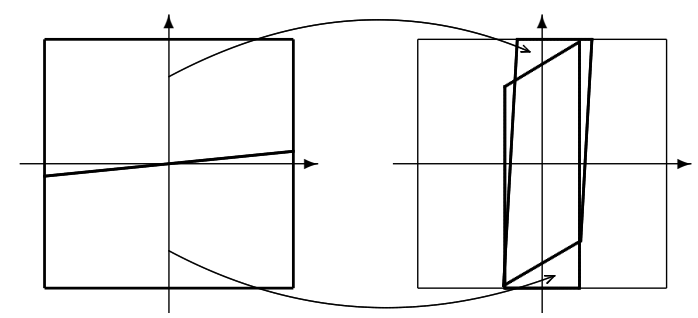

Fig. 2. A picture of $f$ with $\rho=0.1, \psi_{1}=\psi_{2}=0, \gamma=1.8, \lambda=0.3, k=0.1, a_{1}=a_{2}=0$ and $-b_{1}=b_{2}=0.8$

The case $\rho \neq 0, k=\psi_{1}=\psi_{2}=0$ and $\gamma=2$ is treated by Falconer in [5]. He proved that for almost all parameters $\gamma$ and $\lambda$, the dimension satisfies $\operatorname{dim}_{\mathrm{H}} \Lambda=1-\log \gamma / \log \lambda$.

The case $k=0$ and $\gamma=2$ is covered by Simon's paper [13. He proved equality for all parameters. We prove that we have equality for all parameters, also when $k, \psi_{1}$ and $\psi_{2}$ are not nessesarily zero. More precisely, we use Theorem 1 to prove the following theorem. 
Theorem 3. If $a_{1}, a_{2},-b_{1}=b_{2}=\gamma-1$ and

$$
(\gamma, \lambda, k, \rho) \in\{(\gamma, \lambda, k, \rho): \gamma>2 \lambda, \rho \neq 0\}
$$

are numbers such that $f: K \backslash N \rightarrow K$, then $f: K \backslash N \rightarrow K$ defined by (3) has an attractor $\Lambda$ with dimension

$$
\operatorname{dim}_{\mathrm{H}} \Lambda=\min \{2,1-\log \gamma / \log \lambda\} .
$$

Let $\psi_{1}=\psi_{2}=0,1<\gamma<2,0<\lambda<1, a_{1}=a_{2}=0$ and $b_{1}=-b_{2}=$ $1-\gamma$. Then if $\rho=0$, the attractor is $\Lambda=\left\{\left(x_{1}, x_{2}\right): x_{1}=0,\left|x_{2}\right| \leq \gamma-1\right\}$, and so $\operatorname{dim}_{\mathrm{H}} \Lambda=1$. If $\rho \neq 0$ and $\gamma>2 \lambda$ then the dimension $\operatorname{dim}_{\mathrm{H}} \Lambda$ is given by (4). The dimension can be made arbitrarily close to 2 by choosing $\lambda$ close to 1 . Then the dimension is bounded away from 1 for any $\rho \neq 0$, but it is 1 for $\rho=0$.

Proof of Theorem 3. It is clear from Theorem 2 that $f$ has zero multiplicity entropy if $k \neq 0$. If $k=0$ then the multiplicity entropy is trivially zero.

We claim that if $\gamma>2 \lambda$ and $\rho \neq 0$ then $f$ satisfies condition (T). Let us prove this claim. It is clear that the cone spanned by the vectors

$$
\left(\frac{-\rho_{\psi}}{\gamma-\lambda}, 1\right) \text { and }\left(\frac{\rho+\rho_{\psi}}{\gamma-\lambda}, 1\right)
$$

defines an unstable cone family at any point of $K \backslash N$. Denote this cone by $C^{\mathrm{u}}$.

Let $\sigma_{1} \subset K \cap\left\{x_{2}>k x_{1}\right\}$ and $\sigma_{2} \subset K \cap\left\{x_{2}<k x_{1}\right\}$ be two curves such that if $v_{1}$ and $v_{2}$ are two tangent vectors of the curves, then $v_{1}, v_{2} \in C^{\mathrm{u}}$. The vectors $v_{1}$ and $v_{2}$ are mapped by $d_{x} f$ to

$$
u_{1}=\left[\begin{array}{ll}
\lambda & \rho+\psi_{1}\left(x_{2}\right) \\
0 & \gamma
\end{array}\right] v_{1} \quad \text { and } \quad u_{2}=\left[\begin{array}{ll}
\lambda & \psi_{2}\left(x_{2}\right) \\
0 & \gamma
\end{array}\right] v_{2}
$$

respectively. One checks that $u_{1}$ is contained in the cone spanned by

$$
\left(-\rho_{\psi} \frac{\lambda}{\gamma(\gamma-\lambda)}+\frac{\rho-\rho_{\psi}}{\gamma}, 1\right) \text { and } \quad\left(\left(\rho+\rho_{\psi}\right) \frac{\lambda}{\gamma(\gamma-\lambda)}+\frac{\rho+\rho_{\psi}}{\gamma}, 1\right)
$$

and $u_{2}$ is contained in the cone spanned by

$$
\left(-\rho_{\psi} \frac{\lambda}{\gamma(\gamma-\lambda)}+\frac{-\rho_{\psi}}{\gamma}, 1\right) \text { and }\left(\left(\rho+\rho_{\psi}\right) \frac{\lambda}{\gamma(\gamma-\lambda)}+\frac{\rho_{\psi}}{\gamma}, 1\right)
$$

The intersection of these two cones is trivial if

$$
-\rho_{\psi} \frac{\lambda}{\gamma(\gamma-\lambda)}+\frac{\rho-\rho_{\psi}}{\gamma}>\left(\rho+\rho_{\psi}\right) \frac{\lambda}{\gamma(\gamma-\lambda)}+\frac{\rho_{\psi}}{\gamma},
$$

or equivalently, if $\gamma>2 \lambda$. This proves the claim. 
By Corollary 1 it now follows that

$$
\operatorname{dim}_{\mathrm{H}} \Lambda=1-\frac{\log \gamma}{\log \lambda},
$$

unless $\log \gamma+\log \lambda>0$, in which case $\operatorname{dim}_{\mathrm{H}} \Lambda=2$.

Let us end this section by considering the attractor of the map in Figure 2 The dimension of the attractor is

$$
\operatorname{dim}_{\mathrm{H}} \Lambda=1.488 \ldots
$$

There is a picture of the attractor $\Lambda$ in Figure 3 .

We may also consider the dimension of $\Lambda$ when $\gamma=1.8, \lambda=0.5$ and $k=0.1$. Then

$$
\operatorname{dim}_{\mathrm{H}} \Lambda=1.848 \ldots
$$

A picture of this attractor is in Figure 4 . Both pictures were drawn by calculating the iterates of a small curve with tangents in the unstable cones.

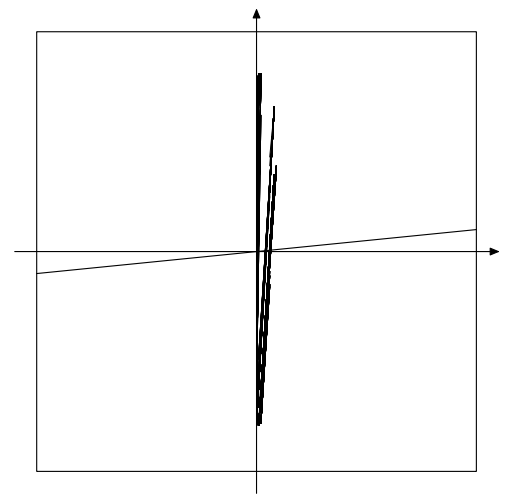

Fig. 3. Attractor of the map in Fig. 2

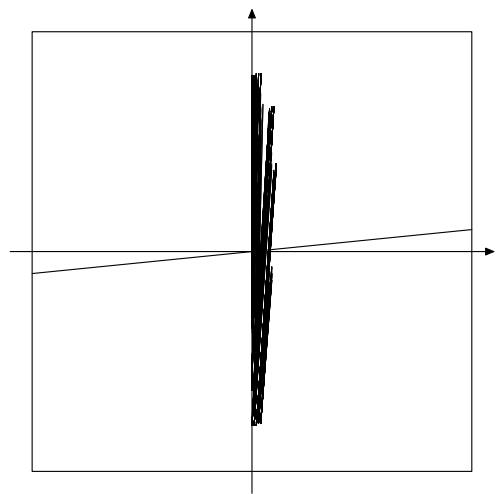

Fig. 4. Attractor of the map in Fig. 2 but with $\lambda=0.5$.

8. Proof of Theorem 1. Assume that $f$ satisfies the conditions (A1)(A4) and condition (T) with $\left(\varepsilon_{0}, \delta\right)$-intersections. Let $\varepsilon>0$.

8.1. Coding of the system. We introduce a coding of the system $f: \Lambda \rightarrow \Lambda$. If $x \in \Lambda$ then there is a sequence $\underline{s}(x)=\left\{i_{k}\right\}_{k \in \mathbb{N} \cup\{0\}}$ such that $f^{k}(x) \in K_{i_{k}}$ for every $k \in \mathbb{N} \cup\{0\}$. We let $\Sigma=\Sigma(\Lambda)$ be the set of all such sequences, that is, $\Sigma(\Lambda)=\underline{s}(D)$.

Given a sequence $\underline{a}=\left\{a_{i}\right\}_{i \in \mathbb{N} \cup\{0\}}$, we define the cylinder ${ }_{k}[\underline{a}]_{l}$ by

$$
{ }_{k}[\underline{a}]_{l}:=\left\{\underline{b}=\left\{b_{i}\right\}_{i} \in \Sigma: b_{i}=a_{i}, \forall i=k, k+1, \ldots, l\right\} .
$$

For any cylinder $k[\underline{a}]_{l}$, there is a corresponding set

$$
\left\{x \in D:\left\{i_{n}\right\}_{n \in \mathbb{N} \cup\{0\}}=\underline{s}(x), i_{n}=a_{n}, \forall n=k, k+1, \ldots, l\right\} .
$$


We denote this set by $\rho\left({ }_{k}[\underline{a}]_{l}\right)$. The sets $\left.\rho_{k}[\underline{a}]_{l}\right)$ will also be called cylinders. A set of the form ${ }_{0}[\underline{a}]_{n-1}$ or $\rho\left({ }_{0}[\underline{a}]_{n-1}\right)$ will be called an $n$-cylinder.

8.2. Images of curves. In this section we make use of condition A4, that the multiplicity entropy is zero, to get some estimates.

For $r \in \mathbb{N}$, we let $\mathcal{D}_{r}(\varepsilon)$ be the set of $r$-cylinders $\rho\left({ }_{0}[\underline{a}]_{r-1}\right)$ such that there exists a point $p \in \rho\left({ }_{0}[\underline{a}]_{r-1}\right)$ with

$$
\begin{array}{ll}
e^{\left(\chi_{\mathrm{u}}-\varepsilon\right) r}\|v\| \leq\left\|d_{p}\left(f^{r}\right)(v)\right\| \leq e^{\left(\chi_{\mathrm{u}}+\varepsilon\right) r}\|v\|, & \forall v \in C^{\mathrm{u}}(p), \\
e^{\left(\chi_{\mathrm{s}}-\varepsilon\right) r}\|v\| \leq\left\|\left(d_{p} f^{r}\right)^{-1}(v)\right\| \leq e^{\left(\chi_{\mathrm{s}}+\varepsilon\right) r}\|v\|, & \forall v \in C^{\mathrm{s}}\left(f^{r}(p)\right) .
\end{array}
$$

Let $q, r \in \mathbb{N}, l>0$ and let $\gamma$ be a curve of length $l$ with tangents in the unstable cones. Let $\mathcal{W}_{0}=\{\gamma\}$. We define $\mathcal{W}_{n}$ inductively. If $\mathcal{W}_{n-1}$ is a collection of curves, then we let $\mathcal{W}_{n}$ be the set of curves that are connected pieces of length between $l$ and $2 l$, contained in the union of $\mathcal{D}_{r}(\varepsilon)$ and in some $f^{q}(\sigma), \sigma \in \mathcal{W}_{n-1}$.

Since we require that the lengths of the curves in $\mathcal{W}_{n}$ are between $l$ and $2 l$, the set $\mathcal{W}_{n}$ might not be uniquely defined, since there are several ways to divide a curve of length larger than $2 l$ into pieces of length between $l$ and $2 l$. It is however not important how this is done, so we will not give a precise definition of $\mathcal{W}_{n}$. For instance, one way of doing this is to begin at one end of the curve, and cut off pieces of length $l$ and put them in $\mathcal{W}_{n}$. As long as the remaining part is longer than $2 l$ we cut off another piece of length $l$. This process will stop when we have a remaining piece of length between $l$ and $2 l$. Putting this remaining piece into $\mathcal{W}_{n}$, we will have achieved what we want.

Lemma 1. Let $f: K \backslash N \rightarrow K$ satisfy conditions A1)-(A4). For any $\varepsilon>0$, there exist constants $C, q, r, l>0$, and a curve $\gamma$ with tangents in the unstable cones, such that if $N(n)$ denotes the number of curves in $\mathcal{W}_{n}$, then

$$
C^{-1} e^{\left(\chi_{\mathrm{u}}-\varepsilon\right) q(n-k)} \leq \frac{N(n)}{N(k)} \leq C e^{\left(\chi_{\mathrm{u}}+\varepsilon\right) q(n-k)},
$$

for all $n \geq k \geq 1$, and the derivatives of $f^{q k}, k \in \mathbb{N}$, at a point $p \in W \in \mathcal{W}_{n}$ satisfy

$$
\begin{aligned}
C^{-1} e^{\left(\chi_{\mathrm{u}}-\varepsilon\right) q k}\|u\| & \leq\left\|d_{p}\left(f^{q k}\right)(u)\right\| \leq C e^{\left(\chi_{\mathrm{u}}+\varepsilon\right) q k}\|u\|, \\
C^{-1} e^{-\left(\chi_{\mathrm{s}}+\varepsilon\right) q k}\|v\| & \leq\left\|\left(d_{p} f^{q k}\right)^{-1}(v)\right\| \leq C e^{-\left(\chi_{\mathrm{s}}-\varepsilon\right) q k}\|v\|,
\end{aligned}
$$

for all $u \in C^{\mathrm{u}}(p)$ and $v \in C^{\mathrm{s}}\left(f^{q k}(p)\right)$.

Proof. Since the multiplicity entropy is zero, we can take $q$ so large and $l>0$ so small that any curve of length $l$ with tangents in the unstable cone is cut into at most $e^{\varepsilon q}$ pieces when mapped by $f^{q}$.

Since the Lebesgue measure of the complement of the union of $\mathcal{D}_{r}(\varepsilon)$ vanishes as $r \rightarrow \infty$, we can choose $r$ so large that the Lebesgue measure of the union of $\mathcal{D}_{r}(\varepsilon)$ is as close to that of $K$ as we like. Using (A3) and (A4), 
we see that it is even possible to choose $r$ so large that the intersection of the complement of the union of $\mathcal{D}_{r}(\varepsilon)$ with any curve of length at least $l$ with tangents in the unstable cone has one-dimensional Lebesgue measure as small as we like.

Hence by first choosing $q$ and $l$, and then $r$ depending on $l$, it is possible to achieve that the sum of the lengths of the curves in $\mathcal{W}_{n}$ satisfies

$$
C_{0} e^{\left(\chi_{\mathrm{u}}-\varepsilon\right) q n} \leq \sum_{\sigma \in \mathcal{W}_{n}} \operatorname{length}(\sigma) \leq C_{0} e^{\left(\chi_{\mathrm{u}}+\varepsilon\right) q n},
$$

where $C_{0}$ is a constant depending on $f, q, l$ and $r$. This implies that the number of curves in $\mathcal{W}_{n}$ satisfies the statement in the lemma.

8.3. Frostman's lemma. We define a probability measure $\mu_{n}$ with support on $\bigcup \mathcal{W}_{n}$ by

$$
\mu_{n}=\frac{1}{N(n)} \sum_{W \in \mathcal{W}_{n}} \nu_{W},
$$

where $\nu_{W}$ denotes the normalised Lebesgue measure on the curve $W$, and $N(n)$ denotes the number of elements in $\mathcal{W}_{n}$ as in Lemma 1.

By taking a subsequence we can achieve that $\mu_{n}$ converges weakly to a probability measure $\mu$ with support in $\Lambda$. This measure will not be invariant, but its conditional measures on the unstable manifold will be absolutely continuous with respect to the corresponding conditional measures of the SRB-measure, almost surely.

We will use the following method, originating from Frostman [6], to estimate the dimension of $\Lambda$. If

$$
\iint \frac{d \mu(x) d \mu(y)}{|x-y|^{s}}<\infty
$$

then $\operatorname{dim}_{\mathrm{H}} \Lambda \geq \operatorname{dim}_{\mathrm{H}} \operatorname{supp} \mu \geq s$. For a proof of this, see Falconer's book [4].

Let $M$ be a number. Then

$$
\begin{aligned}
\iint \min \left\{M, \frac{1}{|x-y|^{s}}\right\} & d \mu_{n}(x) d \mu_{n}(y) \\
& \rightarrow \iint \min \left\{M, \frac{1}{|x-y|^{s}}\right\} d \mu(x) d \mu(y) \quad \text { as } n \rightarrow \infty,
\end{aligned}
$$

and

$$
\begin{aligned}
\iint \min \left\{M, \frac{1}{|x-y|^{s}}\right\} d \mu(x) & d \mu(y) \\
& \rightarrow \iint \frac{1}{|x-y|^{s}} d \mu(x) d \mu(y) \quad \text { as } M \rightarrow \infty .
\end{aligned}
$$

We will therefore estimate

$$
E_{s}(n, M)=\iint \min \left\{M, \frac{1}{|x-y|^{s}}\right\} d \mu_{n}(x) d \mu_{n}(y) .
$$


It is clear that $E_{s}(n, M) \leq M$. By the definition of the measure $\mu_{n}$ we immediately get

$$
E_{s}(n, M)=\sum_{W, V \in \mathcal{W}_{n}} \frac{1}{N(n)^{2}} \iint \min \left\{M, \frac{1}{|x-y|^{s}}\right\} d \nu_{V}(x) d \nu_{W}(y)
$$

We rewrite (7) as

with

$$
E_{s}(n, M)=J_{1}+J_{2}
$$

$$
\begin{aligned}
& J_{1}=\sum_{W \in \mathcal{W}_{n}} \frac{1}{N(n)^{2}} \iint \min \left\{M, \frac{1}{|x-y|^{s}}\right\} d \nu_{W}(x) d \nu_{W}(y), \\
& J_{2}=\sum_{\substack{W, V \in \mathcal{W}_{n} \\
V \neq W}} \frac{1}{N(n)^{2}} \iint \min \left\{M, \frac{1}{|x-y|^{s}}\right\} d \nu_{V}(x) d \nu_{W}(y) .
\end{aligned}
$$

To estimate $J_{1}$ we note that

Hence

$$
\iint \min \left\{M, \frac{1}{|x-y|^{s}}\right\} d \nu_{W}(x) d \nu_{W}(y) \leq M
$$

and so $J_{1} \rightarrow 0$ as $n \rightarrow \infty$.

$$
J_{1} \leq \sum_{W \in \mathcal{W}_{n}} \frac{M}{N(n)^{2}}=\frac{M}{N(n)}
$$

We will now estimate $J_{2}$ and show that $J_{2}$ is bounded as $n \rightarrow \infty$, provided that $s$ is sufficiently small.

Let $m<n$ and $W \in \mathcal{W}_{n}$. Then there is a unique $\alpha \in \mathcal{W}_{n-m}$ such that $W \subset f^{q m}(\alpha)$. Consider the set $W_{-m} \subset \alpha$ such that $W=f^{q m}\left(W_{-m}\right)$.

Fix $m<n$ and take two different $\alpha$ and $\beta$ in $\mathcal{W}_{n-m}$ such that $\alpha_{-1}$ and $\beta_{-1}$ are in different cylinders. By condition (T) this implies that $\alpha$ and $\beta$ intersect $\left(\varepsilon_{0}, \delta\right)$-transversally. We will consider all manifolds $W$ and $V$ in $\mathcal{W}_{n}$ such that $W_{-m} \subset \alpha, V_{-m} \subset \beta$, and $W_{-m}$ and $V_{-m}$ are in the same $q m$-cylinder, denoted by $S_{m}\left(W_{-m}\right)$. There is a picture of this in Figure 5 .

Note that $W$ and $V$ intersect if and only if $W_{-m}$ and $V_{-m}$ intersect, since $W_{-m}$ and $V_{-m}$ are in the same $q m$-cylinder. If $W_{-m} \subset \alpha$ intersects $\beta$, then

$$
\sum_{\substack{V \in \mathcal{W}_{n} \\ V_{-m} \subset \beta \cap S_{m}\left(W_{-m}\right)}} \iint \frac{1}{|x-y|^{s}} d \nu_{V}(x) d \nu_{W}(y) \leq C_{1} e^{\left(\chi_{\mathrm{u}}-\chi_{\mathrm{s}}+2 \varepsilon\right)(s-1) q m},
$$

where $C_{1}$ does not depend on $W, \alpha$ and $\beta$. Indeed, if $m$ is large, then we may assume that $W_{-m}$ and $V_{-m} \subset \beta \cap S_{m}\left(W_{-m}\right)$ are contained in a ball of radius $\varepsilon_{0}$, and so the manifolds $f^{q m}(\beta)$ and $W$ intersect $\left(\varepsilon_{0}, C^{-2} e^{\left(-\chi_{\mathrm{u}}+\chi_{\mathrm{s}}-2 \varepsilon\right) q m} \delta\right)$ transversally, so that

$$
\sum_{\substack{V \in \mathcal{W}_{n} \\ C \beta \cap S_{m}\left(W_{-m}\right)}} \iint \frac{1}{|x-y|^{s}} d \nu_{V}(x) d \nu_{W}(y) \leq C_{0} \iint_{\gamma_{1}} \frac{1}{\gamma_{2}} \frac{1}{|x-y|^{s}} d x d y
$$




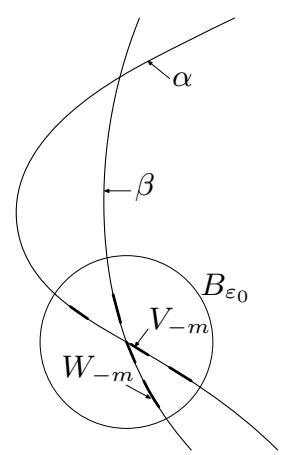

Fig. 5. A picture of intersections of unstable manifolds. The pre-images $W_{-m}$ and $V_{-m}$ are the thicker segments.

where $\gamma_{1}$ and $\gamma_{2}$ are the curves

$$
\begin{aligned}
& \gamma_{1}=\left\{\left(x_{1}, x_{2}\right): x_{1}=0,\left|x_{2}\right|<\operatorname{diam} K\right\}, \\
& \gamma_{2}=\left\{\left(x_{1}, x_{2}\right):\left|x_{2}\right|<l, x_{2}=C^{2} e^{\left(\chi_{\mathrm{u}}-\chi_{\mathrm{s}}+2 \varepsilon\right) q m} \delta^{-1} x_{1}\right\},
\end{aligned}
$$

and $C_{0}$ is a constant that depends only on the second derivative of the map and the constants $\operatorname{diam} K$ and $l$. To prove (8), one easily checks that there exists a constant $C_{1}$ such that

$$
C_{0} \iint_{\gamma_{1} \gamma_{2}} \frac{1}{|x-y|^{s}} d x d y \leq C_{1} e^{\left(\chi_{\mathrm{u}}-\chi_{\mathrm{s}}+2 \varepsilon\right)(s-1) q m} .
$$

We now consider those manifolds $W$ such that $W_{-m} \subset \alpha$ does not intersect $\beta$. First, we consider those $V$ such that $W_{-m}$ and $V_{-m}$ lie in some ball $B_{\varepsilon_{0}}$ in the spirit of (T). If the distance between $W_{-m}$ and $\beta$ is $d\left(W_{-m}, \beta\right)$, then the distance between $W$ and $V \subset f^{q m}(\beta)$ is larger than $C^{-1} e^{\left(\chi_{s}-\varepsilon\right) m} d\left(W_{-m}, \beta\right)$ by (6). If we choose the length $l$ in the construction of $\mathcal{W}_{n}$ sufficiently small, then we can approximate the integral by

$$
\sum_{\substack{V \in \mathcal{W}_{n} \\ \subset \in \cap S_{m}\left(W_{-m}\right)}} \iint \frac{1}{|x-y|^{s}} d \nu_{V}(x) d \nu_{W}(y) \leq l^{-2} \iint_{\gamma_{1} \gamma_{2}} \frac{1}{|x-y|^{s}} d x d y,
$$

where $\gamma_{1}$ and $\gamma_{2}$ are two parallel line segments of lengths $l$ and diam $K$, and with distance $d\left(W_{-m}, \beta\right) / 2$. The last integral is estimated by

$$
\begin{aligned}
\iint_{\gamma_{1} \gamma_{2}} \frac{1}{|x-y|^{s}} d x d y & \leq \int_{-\infty}^{\infty} \frac{1}{\left(\sqrt{x^{2}+\left(d\left(W_{-m}, \beta\right) / 2\right)^{2}}\right)^{s}} d x \\
& =2^{s} e^{\left(\chi_{s}-\varepsilon\right)(1-s) q m} d\left(W_{-m}, \beta\right)^{1-s} \int_{0}^{\infty} \frac{\mathrm{d} x}{\left(1+x^{2}\right)^{s / 2}},
\end{aligned}
$$


and so

$$
\begin{aligned}
& \iint \frac{1}{|x-y|^{s}} d \nu_{V}(x) d \nu_{W}(y) \\
& \leq C_{2} e^{\left(\chi_{s}-\varepsilon\right)(1-s) q m} d\left(W_{-m}, \beta\right)^{1-s}
\end{aligned}
$$

for some constant $C_{2}$, provided that $s>1$.

We cover the intersections of $\alpha$ and $\beta$ by balls $B_{\varepsilon_{0}}$. (Actually we cover the parts $\alpha$ and $\beta$ where $\alpha$ and $\beta$ are within distance $\varepsilon_{0}$ of each other.) Since $K$ is a bounded set, the number of such balls will always be less than some number $N_{B}$. A manifold $W_{-m} \subset \alpha$ either intersects one of these balls or lies a distance at least $\varepsilon_{0}$ from each of the intersections of $\alpha$ and $\beta$.

If $W_{-m}$ lies in $B_{\varepsilon_{0}}$, with distance $d_{W_{-m}}$ from the centre of the ball, then by property (T), the distance between $W_{-m}$ and $V_{-m} \subset \beta$ is at least $\delta d_{W_{-m}}$. The manifolds $W_{-m}$ and $V_{-m}$ are subsets of the two larger manifolds $\alpha$ and $\beta$ (see Figure 5). On each side of the intersection of these larger manifolds (or, in case they do not intersect, on each side of a point on each curve that are closest to the other curve) we can enumerate the pairs $W_{-m}$ and $V_{-m}$, such that the distance from the centre of the ball $B_{\varepsilon_{0}}$ to the $i$ th manifold $W_{-m}$ is increasing.

Since two different $W_{-m}$ do not intersect, the distance from the centre of $B_{\varepsilon_{0}}$ to the $i$ th manifold $W_{-m}$ is at least $i l /\left(C e^{\left(\chi_{\mathrm{u}}+\varepsilon\right) q m}\right)$, since the length of each $W_{-m}$ is at least $l /\left(C e^{\left(\chi_{\mathrm{u}}+\varepsilon\right) q m}\right)$ by (5). (We measure the distance along the large manifold containing all the $W_{-m}$.) Hence the distance between the $i$ th $W_{-m}$ and $V_{-m} \subset \beta$ is at least $\delta i l /\left(C e^{\left(\chi_{\mathrm{u}}+\varepsilon\right) q m}\right)$ and so the distance between the corresponding $W$ and $V$ is at least $C^{-2} e^{\left(\chi_{s}-\varepsilon\right) q m} \delta i l / e^{\left(\chi_{\mathrm{u}}+\varepsilon\right) q m}$.

Since the length of $W_{-m}$ is at least $l /\left(C e^{\left(\chi_{\mathrm{u}}+\varepsilon\right) q m}\right)$, there can be at most $C_{3} e^{\left(\chi_{\mathrm{u}}+\varepsilon\right) q m}$ different $W_{-m}$ in $B_{\varepsilon_{0}}$, where $C_{3}$ is a constant that depends on $l$ and $\varepsilon_{0}$. By (8) and (9),

$$
\begin{gathered}
\sum_{\substack{W-m \subset \alpha \cap B_{\varepsilon_{0}} \\
V_{-m} \subset \beta \cap S_{m}\left(W_{-m}\right) \cap B_{\varepsilon_{0}}}} \iint \frac{1}{|x-y|^{s}} d \nu_{V}(x) d \nu_{W}(y) \\
<C_{1} e^{\left(\chi_{\mathrm{u}}-\chi_{\mathrm{s}}+2 \varepsilon\right)(s-1) q m}+2 \sum_{i=1}^{C_{3} e^{\left(\chi_{\mathrm{u}}+\varepsilon\right) q m}} C_{2}\left(C^{-2} e^{\left(\chi_{\mathrm{s}}-\varepsilon\right) q m} \delta i \frac{l_{0}}{e^{\left(\chi_{\mathrm{u}}+\varepsilon\right) q m}}\right)^{1-s} \\
<C_{4} e^{\left(\chi_{\mathrm{u}}-\chi_{\mathrm{s}}+2 \varepsilon\right)(s-1) q m}\left(e^{\left(\chi_{\mathrm{u}}+\varepsilon\right) q m}\right)^{2-s} .
\end{gathered}
$$

If we sum over the balls $B_{\varepsilon_{0}}$ needed to cover the intersection of $\alpha$ and $\beta$, we get 


$$
\begin{aligned}
\sum_{B_{\varepsilon_{0}}} & \sum_{\substack{W-m \subset \alpha \cap B_{\varepsilon_{0}} \\
V_{-m} \subset \beta \cap S_{m}\left(W_{-m}\right) \cap B_{\varepsilon_{0}}}} \iint \frac{1}{|x-y|^{s}} d \nu_{V}(x) d \nu_{W}(y) \\
& <C_{5} e^{\left(\chi_{\mathrm{u}}-\chi_{\mathrm{s}}+2 \varepsilon\right)(s-1) q m}\left(e^{\left(\chi_{\mathrm{u}}+\varepsilon\right) q m}\right)^{2-s} .
\end{aligned}
$$

For those $W$ and $V$ such that $W_{-m}$ and $V_{-m}$ are not inside a ball $B_{\varepsilon_{0}}$ we have $d(W, V)>C^{-1} e^{\left(\chi_{\mathrm{s}}-\varepsilon\right) q m} \varepsilon_{0}$, and by $(9)$,

$$
\begin{aligned}
& \iint \frac{1}{|x-y|^{s}} d \nu_{V}(x) d \nu_{W}(y) \\
& \leq C_{2} e^{\left(\chi_{\mathrm{s}}-\varepsilon\right)(1-s) q m} C^{s-1} \varepsilon_{0}^{1-s}=C_{6} e^{\left(\chi_{\mathrm{s}}-\varepsilon\right)(1-s) q m} .
\end{aligned}
$$

Since the length of each $W_{-m}$ and $V_{-m}$ is at least $l /\left(C e^{\left(\chi_{\mathrm{u}}+\varepsilon\right) q m}\right)$ by (5), and $W \subset \alpha$ and $V \subset \beta$, where $\alpha$ and $\beta$ are fixed, there is a constant $\bar{C}_{7}$, independent of $m, n, \alpha$ and $\beta$, such that the number of such pairs $W$ and $V$ satisfying $\sqrt{12}$ is at most $C_{7} e^{\left(\chi_{\mathrm{u}}+\varepsilon\right) q m}$.

By (11) and (12) we get

$$
\sum_{\substack{W, V \in \mathcal{W}_{n} \\ W-m \subset \alpha \\ V_{-m} \subset \beta}} \iint \frac{1}{|x-y|^{s}} d \nu_{V}(x) d \nu_{W}(y)
$$

$<C_{5} e^{\left(\chi_{\mathrm{u}}-\chi_{\mathrm{s}}+2 \varepsilon\right)(s-1) m}\left(e^{\left(\chi_{\mathrm{u}}+\varepsilon\right) q m}\right)^{2-s}+C_{6} C_{7} e^{\left(-\chi_{\mathrm{s}}+\varepsilon\right)(s-1) q m} e^{\left(\chi_{\mathrm{u}}+\varepsilon\right) q m}$.

We will now sum over all $m, \alpha$ and $\beta$, and write $J_{2}$ as $J_{2}=J_{3}+J_{4}$ with

$$
\begin{aligned}
J_{3} & =\sum_{m=0} \sum_{\substack{\alpha, \beta \in \mathcal{W}_{n-m} \\
\alpha \neq \beta}} \sum_{\substack{W, V \in \mathcal{W}_{n} \\
W-m \subset \alpha \\
V_{-m} \subset \beta}} \frac{\iint \min \left\{M, 1 /|x-y|^{s}\right\} d \nu_{V}(x) d \nu_{W}(y)}{N(n)^{2}}, \\
J_{4} & =\sum_{m=0}^{n-1} \sum_{\alpha \in \mathcal{W}_{n-m}} \sum_{\substack{W, V \in \mathcal{W}_{n} \\
W_{-m}, V_{-m} \subset \alpha \\
W_{-m} \neq V_{-m}}} \frac{\iint \min \left\{M, 1 /|x-y|^{s}\right\} d \nu_{V}(x) d \nu_{W}(y)}{N(n)^{2}} .
\end{aligned}
$$

As for $J_{1}$, we obtain $J_{4} \rightarrow 0$ as $n \rightarrow \infty$. It remains to estimate $J_{3}$.

Using that there are $N(n-m)$ different $\alpha$ and $\beta$, we get, by 10 and (13),

$$
\begin{aligned}
J_{3} \leq & \sum_{m=0}^{n-1} N(n-m)^{2} \frac{C_{5} e^{\left(\chi_{\mathrm{u}}-\chi_{\mathrm{s}}+2 \varepsilon\right)(s-1) q m}\left(e^{\left(\chi_{\mathrm{u}}+\varepsilon\right) q m}\right)^{2-s}}{N(n)^{2}} \\
& +\sum_{m=0}^{n-1} N(n-m)^{2} \frac{C_{6} C_{7} e^{\left(-\chi_{\mathrm{s}}(s-1)+\varepsilon(s-1)\right) q m} e^{\left(\chi_{\mathrm{u}}+\varepsilon\right) q m}}{N(n)^{2}} .
\end{aligned}
$$


Letting $C_{8}=C_{5}+C_{6} C_{7}$ yields

$$
J_{3} \leq C_{8} \sum_{m=0}^{n-1} \frac{N(n-m)^{2} e^{\left(\chi_{\mathrm{u}}-\chi_{\mathrm{s}}(s-1)+s \varepsilon\right) q m}}{N(n)^{2}},
$$

and $C_{8}$ does not depend on $n$. By Lemma 1 we have $N(n-m) / N(n) \leq$ $C e^{-\left(\chi_{\mathrm{u}}-\varepsilon\right) q m}$, so

$$
J_{3} \leq C_{8} \sum_{m=0}^{n-1} e^{\left(-\chi_{\mathrm{u}}-\chi_{\mathrm{s}}(s-1)+\varepsilon(s+2)\right) q m} .
$$

We conclude that $J_{3}$ is bounded as a function of $n$ provided that $-\chi_{\mathrm{u}}-$ $(s-1) \chi_{\mathrm{s}}+(s+2) \varepsilon<0$ and $s<2$, or equivalently

$$
s<1-\frac{\chi_{\mathrm{u}}-3 \varepsilon}{\chi_{\mathrm{s}}-\varepsilon} \text { and } s<2 .
$$

We have thus proved that if $s$ satisfies (14), then $J_{1}$ and $J_{2}=J_{3}+J_{4}$ are bounded, and so the integral

$$
\iint \min \left\{M, \frac{1}{|x-y|^{s}}\right\} d \mu(x) d \mu(y)
$$

is uniformly bounded and hence converges as $M \rightarrow \infty$. This proves that

$$
\iint \frac{1}{|x-y|^{s}} d \mu(x) d \mu(y)<\infty
$$

provided that (14) holds. Hence

$$
\operatorname{dim}_{\mathrm{H}} \Lambda \geq \min \left\{2,1-\frac{\chi_{\mathrm{u}}-3 \varepsilon}{\chi_{\mathrm{s}}-\varepsilon}\right\} .
$$

Now let $\varepsilon \rightarrow 0$.

\section{References}

[1] J. C. Alexander and J. A. Yorke, Fat baker's transformations, Ergodic Theory Dynam. Systems 4 (1984), 1-23.

[2] J. Buzzi, Intrinsic ergodicity of affine maps in $[0,1]^{d}$, Monatsh. Math. 124 (1997), 97-118.

[3] P. Erdôs, On a family of symmetric Bernoulli convolutions, Amer. J. Math. 61 (1939), 974-976.

[4] K. Falconer, Fractal Geometry. Mathematical Foundations and Applications, Wiley, Chichester, 1990.

[5] - , The Hausdorff dimension of some fractals and attractors of overlapping construction, J. Statist. Phys. 47 (1987), 123-132.

[6] O. Frostman, Potentiel d'équilibre et capacité des ensembles avec quelques applications à la théorie des fonctions, Meddelanden från Lunds universitets matematiska seminarium, band 3, Lund, 1935.

[7] B. Kruglikov and M. Rypdal, Entropy via multiplicity, Discrete Contin. Dynam. Systems 16 (2006), 395-410. 
[8] T. Persson, A piecewise hyperbolic map with absolutely continuous invariant measure, Dynam. Systems 21 (2006), 363-378.

[9] —, Absolutely continuous invariant measures for some piecewise hyperbolic affine maps, Ergodic Theory Dynam. Systems 28 (2008), 211-228.

[10] Ya. Pesin, Dynamical systems with generalized hyperbolic attractors: hyperbolic, ergodic and topological properties, ibid. 12 (1992), 123-151.

[11] E. Sataev, Invariant measures for hyperbolic maps with singularities, Russian Math. Surveys 47 (1992), 191-251.

[12] J. Schmeling and S. Troubetzkoy, Dimension and invertibility of hyperbolic endomorphisms with singularities, Ergodic Theory Dynam. Systems 18 (1998), 12571282 .

[13] K. Simon, Hausdorff dimension for non-invertible maps, ibid. 13 (1993), 199-212.

[14] B. Solomyak, On the random series $\sum \pm \lambda^{i}$ (an Erdôs problem), Ann. of Math. 142 (1995), 611-625.

[15] M. Tsujii, Fat solenoidal attractors, Nonlinearity 14 (2001), 1011-1027.

Tomas Persson

Institute of Mathematics

Polish Academy of Sciences

Śniadeckich 8

00-956 Warszawa, Poland

E-mail: tomasp@impan.pl

Received 4 May 2009;

in revised form 1 February 2010 